Annuaire suisse de politique de développement

$1 \mid 1981$

Annuaire Suisse - Tiers Monde 1981

\title{
3. Communication
}

\section{Charlotte Feller}

\section{OpenEdition}

Journals

Édition électronique

URL : http://journals.openedition.org/aspd/1099

DOI : 10.4000/aspd.1099

ISSN : 1663-9669

\section{Éditeur}

Institut de hautes études internationales et du développement

\section{Édition imprimée}

Date de publication : 1 janvier 1981

Pagination : 127-144

ISSN : 1660-5934

\section{Référence électronique}

Charlotte Feller, «3. Communication». Annuaire suisse de politique de développement [En ligne], 1 |

1981, mis en ligne le 19 janvier 2013, consulté le 08 septembre 2020. URL : http://

journals.openedition.org/aspd/1099; DOI : https://doi.org/10.4000/aspd.1099 


\section{COMMUNICATION}

\section{BELGRADE : CONFERENCE DE L'UNESCO}

La 21 e Conférence générale de I'UNESCO a eu lieu à Belgrade du 23 septembre au 28 octobre 1980. Elle réunissait les délégués des 153 Etats membres de l'Organisation. Voici ce qu'en dit le Conseil fédéral dans son rapport de gestion : "Comme lors de la 20e session, les questions relatives à l'information et à la communication ont été au centre des débats. La Conférence a notamment approuvé la création d'un Programme international pour le développement de la communication (PIDC), coordonné par un Conseil intergouvernemental de 35 Etats membres. En se dotant ainsi d'un programme opérationnel, I'UNESCO a manifesté sa volonté d'apporter une contribution concrète à la solution des problèmes d'information et de la communication en renforçant les infrastructures des pays en développement dans ce domaine. Son action n'en continuera pas moins d'être orientée vers la définition - en vue de son instauration - d'un nouvel ordre mondial de l'information et de la communication. La Conférence générale a en effet adopté deux nouvelles résolutions favorisant cette orientation, la première sur les suites à donner aux conclusions de la Commission MacBride, la seconde préconisant l'élaboration d'une Déclaration sur l'instauration d'un nouvel ordre mondial de l'information et de la communication."

"La délégation suisse a adopté une attitude ferme. Inflexible quant aux principes de la liberté de presse let d'expression, fidèle à notre préférence pour l'action concrète, elle a réaffirmé la volonté de la Suisse de contribuer, par une assistance pratique, à remédier au déséquilibre existant entre pays industrialisés et pays en développement dans le domaine de la communication. En conséquence, elle n'a pas participé au consensus sur la résolution concernant les suites du Rapport 
MacBride et s'est opposée à la résolution sur l'élaboration d'une nouvelle Déclaration. J Elle s'est en revanche associée au consensus sur le RIDC et elle a présenté une résolution visant à encourager les échanges temporaires de collaborateurs entre les agences d'information des pays industrialisés et des pays en développement, résolution qui fut adoptée à l'unanimité." ... "Pour l'exercice triennal 1981-1983, la Conférence a approuvé un plafond budgétaire s'élevant à 625.374 .000 dollars. Lors du vote, la Suisse s'est abstenue" $(1,2)$.

En plus de ce que dit le rapport de gestion, la Suisse a demandé le vote et elle a été la seule à voter non sur la résolution concernant l'application de la Déclaration sur les médias (Déclaration sur les principes fondamentaux concernant la contribution des organes d'information au renforcement de la paix et de la compréhension internationale, à la promotion des droits de l'homme et à la lutte contre le racisme, l'apartheid et l'incitation à la guerre).

Pour comprendre la position de la Suisse, il est nécessaire d'expliquer la situation et la position des différents groupes de pays.

Les pays du Tiers Monde font remarquer qu'ils se trouvent dans une situation de dépendance en ce qui concerne l'information. Voici un certain nombre de chiffres tirés du Rapport de la Commission internationale d'étude des problèmes de la communication (rapport MacBride). $\jmath^{\prime \prime}$ Le monde reçoit $80 \%$ de ses nouvelles via Londres, New York et Paris" (3), c'est-à-dire par le truchement des quatre grandes agences de presse occidentales : AFP, AP, Reuter et UPI. "Les grandes agences ne consacrent qu'entre 10 et $30 \%$ de leurs nouvelles à la totalité du monde en développement" (4). L'Annuaire statistique des Nations Unies révèle que dans 91 pays du Tiers Monde "30 à $75 \%$ des programmes de télévision - $55 \%$ en moyenne - sont importés" (5). Et pour transmettre leurs informations, les pays industrialisés "utilisent $90 \%$ du spectre de fréquence et de de l'orbite géostationnaire" (6).

Le déséquilibre est à la fois quantitatif et qualitatif. La proportion des mauvaises nouvelles dans l'information venant du Tiers Monde 
est plus élevée que dans celles concernant les pays industrialisés : elles sont consacrées en majeure partie aux catastrophes, aux échecs, aux conflits, aux difficultés ou aux excès. "Les médias transnationaux exercent une influence majeure sur les idées et les opinions, sur les valeurs et les styles de vie, et par conséquent sur l'évolution, pour le meilleur et pour le pire, de toutes les sociétés" (7).

Les pays du Tiers Monde considèrent que ce déséquilibre et l'influence des transnationales aliènent leur identité culturelle. C'est pourquoi, pour contrebalancer ce courant Nord-Sud, ils réclament le développement de leurs propres moyens de communication de masse de manière à pouvoir informer eux-mêmes leur public et le public des pays industrialisés.

Mais à ce clivage Nord-Sud s'ajoute le clivage Est-Ouest. A la "liberté d'information" défendue par les pays industrialisés, les pays de l'Est opposent la "responsabilité du communicateur", c'est-à-dire le respect de la souveraineté de l'Etat dans le domaine de l'information. Or ce concept peut aller de l'auto-censure à la censure, en passant par la pression économique, l'accès à une seule source d'information et le monopole des moyens de communication. Beaucoup de pays du Tiers Monde se rallient à cette norme. Le problème est de savoir si elle est utilisée pour protéger l'identité culturelle ou pour renforcer les droits acquis des pouvoirs en place.

A ces deux revendications Sud et Est, les pays industrialisés opposent donc la liberté d'information du communicateur (élément de la Déclaration universelle des droits de ('homme), le libre accès à plusieurs sources d'information, le droit du public à l'information, et la promotion de la pluralité des opinions.

La Suisse défend la liberté d'information avec plus de vigueur que les grands exportateurs (les Etats-Unis, la Grande-Bretagne et la France) bien que, à part la Radio Suisse Internationale, les exportations suisses dans le domaine de la communication de masse soient insignifiantes. Mais la Suisse reconnaît aussi le déséquilibre de l'information Nord-Sud. Et elle considère que, pour résoudre ce problème, il faut avant tout aider les pays du Tiers Monde à développer leurs propres moyens d'information. Déjà en 1976, à la Conférence de Nairobi, elle avait clairement défini sa ligne de conduite (8). Jusqu'à maintenant, la DDA ne s'est engagée à finan- 
cer que trois projets dans ce domaine, pour un montant total de 1.365 .000 francs.

A Belgrade, la Suisse ne s'est pas associée au consensus concernant les suites à donner au rapport MacBride. Cette résolution contient un certain nombre d'éléments constitutifs d'un nouvel ordre mondial de l'information : "Ce nouvel ordre mondial de l'information et de la communication pourrait reposer, entre autres bases, sur : (i) l'élimination des déséquilibres et des inégalités qui caractérisent la situation actuelle; (ii) l'élimination des effets négatifs de certains monopoles, publics ou privés, et des concentrations excessives; (iii) la suppression des obstacles internes et externes qui s'opposent à une circulation libre et une diffusion plus large ef mieux équilibrée de l'information et des idées; (iv) la pluralité des sources ef des canaux d'iṇformation; ( $v$ ) la liberté des journalistes et de tous les professionnéls des moyens de communication, une liberté inséparable de la responsabilité ;..." (9)

C'est la notion de "liberté des journalistes" telle qu'elle est mentionnée dans ce paragraphe que la Suisse n'a pas acceptée. De plus, la résolution invite les Etats membres à aménager plus justement les tarifs postaux et de télécommunication ainsi que les positions sur les ondes et sur les satellites. La Suisse y oppose le respect des accords internationaux en la matière. Après le consensus, voici ce qu'a déclaré, entre autres, la délégation suisse : "Nous sommes d'avis que la seule voie à poursuivre, dans le cadre de I'UNESCO, consiste à aider les pays en voie de développement à résoudre leurs problèmes d'information et de communication comme nous nous efforçons de le faire" (10).

En ce qui concerne cette aide à la communication, la DDA s'est engagée à participer au financement du magazine africain "Famille et Développement". Sa contribution s'élève à 550.000 francs pour la période 1979 à 1981 (11). En mars 1981, la DDA s'est également engagée à cofinancer deux projets de I'UNESCO. II s'agit de la création de deux journaux régionaux en langue locale, un au Mali, et l'autre en Haute-Volta. Dans le cadre de ces journaux un réseau de correspondants sera créé. Ceux-ci pourront également fournir des informations aux radios locale et nationale. Pour le Mali, la contribution suisse s'élève à 395.000 francs pour 1981-82 
et pour la Haute-Volta à 420.000 francs pour 1981-82 également (12) .

Revenons à Belgrade : la Suisse a également fait une proposition qui "invite le Directeur général à prendre des mesures facilitant l'échange temporaire de collaborateurs entre, d'une part, les agences d'information des pays industrialisés et, d'autre part, les agences existantes ou à créer dans les pays en voie de développement, afin de favoriser une meilleure compréhension réciproque et de permettre un dialogue véritable fondé sur une meilleure connaissance mutuelle" (13). Cette résolution a été adoptée à l'unanimité.

La Suisse a de plus participé au consensus sur la résolution concernant le Programme international pour le développement de la communication (PIDC). Mais elle aurait voulu que les statuts du Conseil intergouvernemental chargé de diriger le Programme mentionnent la liberté d'information.

Quant à la résolution concernant I'application de la Déclaration sur les médias, elle "invite les Etats membres à prendre les mesures nécessaires pour faire connaître encore plus largement la Déclaration à l'opinion publique, aux journalistes et aux autres représentants des mass médias de leur pays" (14). Ici, la Suisse a demandé le vote et elle a été la seule à voter non.

En 1978, elle avait participé au consensus sur la Déclaration et voici ce qu'avait déclaré le délégué suisse en Commission : "Nous sommes hostiles à toute tendance à établir un lien quelconque entre la protection des journalistes et les principes qui sont à la base de la Déclaration, laquelle prescrit aux moyens d'information ce qu'ils doivent faire ou ne pas faire ou leur assigne des finalités qui d'ailleurs comportent l'obligation, même voilée, de publier ou de diffuser telle ou telle information" (15).

La Suisse a également demandé le vote et voté contre la résolution de Belgrade demandant l'élaboration d'une Déclaration tendant à instaurer un nouvel ordre mondial de l'information. Cette résolution se réfère en effet à la Déclaration sur les médias.

La délégation suisse s'est exprimée en ces termes lors de l'ouverture de la 2 le Conférence générale : "Nous formulons ... de sérieuses réserves quant à la notion de 'droits collectifs'. A notre avis, l'accent devrait plutôt porter sur la protection des droits fondamen- 
taux, qui découlent de la dignité attachée à la personne humaine et dont le respect peut être exigé par des moyens juridictionnels. La liberté de pensée, d'expression, le droit à l'éducation peuvent faire l'objet de tels mécanismes de contrôle et la personne peut se voir accorder les moyens d'exiger de l'Etat le respect de ses droits."

"Un droit à la paix et à la sécurité par exemple, si désirable qu'il puisse être dans son contenu concret, ne saurait être efficacement protégé sur le plan des institutions internes. Ce domaine si important pour l'avenir de l'humanité est proprement politique; il relève des relations internationales" (16).

Rappelons que la liberté d'information en Suisse souffre certaines restrictions légales en ce qui concerne la sécurité de l'Etat et les relations avec l'étranger, la pornographie ou la religion (17).

\section{Notes}

1. Rapport du Conseil fédéral sur sa gestion en 1980, p. 27.

2. Pour le texte des résolutions, voir :

UNESCO, Conférence générale, vingt et unième session, Belgrade, 1980, Rapport de la Commission IV, octobre 1980 :

- résolution concernant le Programme international pour le développement de la communication, Annexe et Recommandations, p. 18;

- résolution concernant le Rapport du Directeur général sur les conclusions de la Commission internationale d'étude des problèmes de la communication, Annexe et Recommandations, addendum et corrigendum, p. 1;

- mesures visant l'application de la Déclaration sur les principes fondamentaux concernant la contribution des organes d'information au renforcement de la paix et de la compréhension internationale, à la promotion des droits de l'homme et à la lutte contre le racisme, l'apartheid et l'incitation à la guerre, adoptée par la Conférence générale de I'UNESCO à sa vingtième session, Annexe et Recommandations, p. 73.

UNESCO 21 C/DR 385 Conférence générale, vingt et unième session, Belgrade 1980, point 14 de l'ordre du jour, 
projet de résolution présenté par le Vénézuela, Amendement au Projet de programme et budget pour 1981-1983, 17 octobre 1980 : Etude de la possibilité et de l'opportunité d'élaborer une Déclaration tendant à l'instauration d'un nouvel ordre mondial de l'information et de la communication, en vue de sa présentation à la Conférence générale de I'UNES$\mathrm{CO}$ lors de sa vingt-deuxième session.

UNESCO 21 C/DR 343 Conférence générale, vingt et unième session, Belgrade 1980, point 24 de l'ordre du jour, Projet de résolution présenté par la Suisse, Amendement au Projet de programme et de budget pour 1981-1983, 13 octobre 1980 : Promotion de l'accès aux moyens de communication et d'une participation accrue du public aux réalisations et à la gestion des médias - Programme de participation et de coopération pour le développement.

Pour la position de la Suisse, voir :

Département fédéral des affaires étrangères, 21 e Conférence générale de I'UNESCO, Documentation sur les questions d'information et de communication, déclarations de la délégation suisse.

En 1980, la Commission nationale suisse pour I'UNESCO a nédigé une prise de position sur le Rapport MacBride. La Commission d'experts pour une conception globale des médias (Commission Kopp), qui aurait dû fournir un rapport pour juillet 1980, n'a pas encore terminé son travail.

3. Sean McBride : Voix multiples, un seul monde, Rapport de la Commission internationale d'étude des problèmes de la communication, UNESCO, Paris, 1980, p. 180.

4. Idem pp. 180-181.

5. Idem p. 153.

6. Idem p. 163.

7. Idem p.137.

8. UNESCO : Actes de la Conférence générale, XIXe session, Nairobi 1976, Volume II, tome I, Comptes rendus des débats, Paris 1978, pp. 498-501. 
9. UNESCO, Conférence générale, vingt et unière session, Rapport de la Commission IV, Annexes et Recommandations, addendum et corrigendum, p. 6.

10. Département fédéral des affaires étrangères, 21 e Conférence générale, document cité.

11. DDA fiche de projet, t. 311, Sénégal 17.

12. Martin Obrist : Projekte ländlicher Kommunikation im Sahel, Direktion für Entwicklungszusammenarbeit und humanitäre Hilfe, Nationale Schweizerische UNESCO-Kommission, Generalversammlung, St. Gallen, 6. März 1981.

13. UNESCO : 21 C/DR 343, document cité.

14. UNESCO, Conférence générale, vingt et unième session, Rapport de la Commission IV, Annexes et Recommandations, p. 73.

15. UNESCO : Actes de la Conférence générale, vingtième session, Paris, 24 octobre au 28 novembre 1978, volume 3, Comptes rendus des débats, 29 e session de la Commission IV, Paris 1980, p. 1072.

Le texte de la Déclaration sur les médias se trouve dans idem, volume 1, Résolutions, Paris 1979, pp. 105 et suiv.

16. Département fédéral des affaires étrangères, 21 e Conférence générale de I'UNESCO, Déclaration du chef de la délégation suisse (Courtoisies d'usage au pays hôte et au Président de la Conférence), 6 octobre 1980, p. 7.

17. Code pénal suisse, articles 27, 296, 204 et 261.

\section{CODE DE CONDUITE SUR LE TRANSFERT DE TECHNOLOGIE}

Aucun progrès n'a été fait dans la négociation sur le code international de conduite de transfert de technologie. On a même enregistré un recul à la 4 e session de la Conférence qui s'est tenue à la CNUCED du 23 mars au 10 avril 1981. En effet, le texte de compromis concernant les responsabilités et obligations des 
parties a été remis en question par les 77 et les EtatsUnis.

Voici ce qu'en dit le Conseil fédéral dans son $15 \mathrm{e}$ Rapport sur la politique économique extérieure : "Des positions fondamentalement divergentes subsistent encore, notamment en ce qui concerne la forme à donner au chapitre relatif aux pratiques commerciales restrictives. Les pays en développement voudraient que soit dressée une liste des clauses de contrats de licence qui pourraient être déclarées irrecevables lorsque dans des cas isolés elles ne sont pas approuvées par les autorités du pays de l'acquéreur de licence pour des raisons d'intérêt national. Les pays industrialisés se laissent en revanche inspirer par la pratique du droit en matière de concurrence, notamment par celle des USA, qui, dans certaines circonstances, qualifie certaines restrictions imposées à l'activité des acquéreurs de licence de pratiques commerciales restrictives dommageables. Ils craignent que si l'on restreignait encore davantage la liberté de contracter, cela se traduise par une baisse d'intérêt envers l'octroi de licences et par une entrave au transfert de technologie. Des divergences insurmontables se font jour à propos du chapitre 'droit applicable et règlement de différends'. Les pays en développement voudraient dans toute la mesure du possible rendre applicable le droit du pays acquéreur de licence" (1).

D'autres questions importantes sont toujours en suspens : les Etats participant à la négociation n'ont pas encore décidé dans quelle mesure le code aurait force obligatoire ou non; ils n'ont pas non plus décidé si le code s'appliquera aussi aux transferts entre sociétés-mères et filiales à l'étranger. Face aux pays du Tiers Monde, les pays industrialisés présentent un front uni. Mais au sein du Groupe B, il y a des divergences que rien ne laisse filtrer. On sait très peu de choses sur la position de la Suisse (2).

Le transfert de technologie est défini ainsi par le code: "Le 
transfert de technologie ... est le transfert des connaissances systématiques nécessaires à la fabrication d'un produit, à l'application d'un procédé ou à la prestation d'un service, et ne s'étend pas aux transactions comportant la simple vente ou le simple louage de biens" (3). Le Code donne une liste des transactions concernées : transactions concemant la propriété industrielle, à l'exception des marques de fabrique, de service, et désignations commerciales quand elles ne font pas partie d'une transaction portant sur un transfert de technologie; transactions impliquant communication de savoirfaire et de connaissances techniques spécialisées; communication des renseignements technologiques nécessaires à l'installation, à l'exploitation et au fonctionnement d'usines et de matériel et aux projets clé en main.

Pour 1980, l'ONU a estimé que les dépenses faites par le Tiers Monde pour l'acquisition de technologie étrangère se sont élevées à 12 milliards de dollars et qu'elles augmentaient à un rythme de $30 \%$ par an (4). A titre de comparaison, le déficit de la balance des paiements des pays du Tiers Monde non-producteurs de pétrole est estimé à 72 milliards de dollars pour 1980 (5).

Mais comment mesurer ces transferts de technologie ? Pour les pays industrialisés, l'OCDE a défini certains indicateurs représentant des flux liés au transfert de technologie : les recettes provenant de la technologie (licences, services techniques et commerciaux, ...), les exportations de biens d'équipement, les investissements directs, les crédits à l'exportation et les prêts internationaux. Ces indicateurs représentent des transactions qui "peuvent" donner lieu à des transferts de technologie mais ne disent rien des transferts effectifs (6). Si on applique ces indicateurs à la Suisse, voici ce que donnent les chiffres (voir tableau Indicateurs de transfert de technologie de la Suisse vers les pays du Tiers Monde, p. 137.

Les estimations diverses faites pour la balance des paiements ne donnent aucune indication sur les recettes provenant de la technologie elle-même. Mais on sait que la Suisse est le premier exportateur mondial de brevets par habitant (7). En 1979, 15.650 demandes étrangères de brevets suisses ont été déposées et 2.086 émanaient des pays du Tiers Monde (voir tableau de la page suivante), soit $13 \%$ (8). Différentes évaluations du revenu des brevets et des licences suisses ont été faites. On peut estimer qu'il est de 
DEMANDES DE BREVETS SUISSES DEPOSEES

PAR LES PAYS DU TIERS MONDE EN 1979

\begin{tabular}{|c|c|c|c|}
\hline Brésil & 309 & Tunisie & 16 \\
\hline Argentine & 269 & *Zimbabwé & 16 \\
\hline Yougoslavie & 204 & Irak & 14 \\
\hline Israël & 195 & ${ }^{*}$ Colombie & 13 \\
\hline Mexique & 160 & Equateur & 13 \\
\hline Corée du Sud & 127 & *Cuba & 11 \\
\hline *Inde & 91 & ${ }^{\star}$ Chypre & 11 \\
\hline Vénézuela & 90 & *Zaire & 10 \\
\hline *Philippines & 63 & ${ }^{*}$ Tanzanie & \\
\hline${ }^{\star}$ Hong-Kong & 51 & ${ }^{\star}$ Bangladesh & \\
\hline ^Egypte & 47 & Bolivie & \\
\hline Singapour & 42 & *Bahrein & \\
\hline Turquie & 38 & Malawi & \\
\hline Iran & 29 & *Ghana & \\
\hline *Algérie & 29 & *Ouganda & \\
\hline *Uruguay & 27 & Bahamas & \\
\hline Pakistan & 25 & ${ }^{*}$ Malte & \\
\hline Sri Lanka & 24 & Costa-Rica & \\
\hline $\begin{array}{l}\text { Organisation africaine } \\
\text { de prop. int. OAPI }\end{array}$ & $e_{21}$ & $\begin{array}{l}\text { *Rwanda } \\
\text { Seychelles }\end{array}$ & \\
\hline${ }^{\star}$ Maroc & 19 & Honduras & \\
\hline Indochine & 17 & ${ }^{\star}$ Zambie & \\
\hline * Jamaĩque & 16 & *Pérou & \\
\hline${ }^{*}$ Kenya & 16 & *Sierre Leone & \\
\hline Syrie & 16 & Total & 2.0 \\
\hline
\end{tabular}

Source : WIPO Industrial Property Statistics 1979, Genève 1981, pp. 11,13 et 15 .

* Pays pour lesquels l'OMPI publie des données par industrie (voir WIPO Industrial Property Statistics 1979, pp. 26-149).

INDICATEURS DE TRANSFERT DE TECHNOLOGIE DE LA SUISSE VERS LES PAYS DU TIERS MONDE

en millions de francs $1978 \quad 1979 \quad 1980$

Exportation de biens d'équip.

$3.620,5 \quad 3.558,8$

$3.825,3$

Investissements directs

$311,2 \cdot 691,5$

590,9

Crédits à l'exportation

$1.484,3 \quad 1.024,6$

299,5

Emprunts sur le marché

$$
2.793,0 \quad 4.454,5 \quad 3.120,8
$$

Flux bancaires, avoirs

$$
\begin{array}{llll}
\text { à long terme } \quad 1.682,6 & 1.621,1 & 159,1
\end{array}
$$

Sources: Statistique annuelle du commerce extérieur de la Suisse, DDA : + 140, + 280, Tableaux statistiques de base concernant l'effort d'aide de la Suisse et des pays membres du Comité d'aide au développement (CAD) de I'OCDE en faveur des pays en développement. 
l'ordre de grandeur de 1 milliard de francs suisses (9). En l'absence de chiffres précis, notons que les exportations de biens d'équipement à destination du Tiers Monde en 1980 s'élevaient à 3,825 milliards de francs, soit $35 \%$ des exportations totales vers le Tiers Monde (10,804 milliards de francs).

Le total des emprunts publics, des flux bancaires et avoirs privés à long terme s'élevait à 3,3 milliards de francs en 1980. Ces chiffres comprennent les capitaux étrangers que les banques suisses acheminent vers le Tiers Monde. A titre de comparaison, le total des investissements de portefeuille bilatéraux et multilatéraux des pays du CAD réunis s'élevait à 24 milliards de dollars, soit 40 milliards de francs en 1980 (10).

C'est en 1972 déjà que des discussions ont commencé sur ce qu'on appelait alors "une nouvelle réglementation internationale du transfert des techniques". Mais c'est à la 4 e Conférence de la CNUCED à Nairobi que les pays du Tiers Monde ont obtenu la rédaction d'un code international de transfert de technologie en même temps qu'ils obtenaient la rédaction d'un code de conduite des transnationales et des règles pour le contrôle des pratiques commerciales restictives. Les négociations proprement dites sur le Code de transfert de technologie ont commencé en octobre 1978.

Les pays du Tiers Monde considèrent que le transfert de technologie est nécessaire à leur industrialisation et qu'il est encore marqué par la persistance de conditions et de pratiques inéquitables. Selon les résolutions de l'Assemblée générale des Nations Unies et de la Conférence de la CNUCED, le code devrait répondre aux besoins et à la situation des pays du Tiers Monde (11).

Quant à la Suisse, voici qu'elle était sa position en 1979, lors de la Conférence des Nations Unies à Vienne sur la science et la technique au service du développement : "Il est et il restera très difficile, pour un pays en développement, de se lancer seul dans la production de nouveaux types de biens, même s'il lui était possible de se procurer une technologie déterminée, et ceci même pour les produits pour lesquels le marché $n$ 'a pas une structure trop oligopolistique qui en rende l'accès presque impossible à des petites entreprises nouvelles." 
... "Les transferts par des entreprises étrangères restent donc un des moyens privilégiés pour un pays en développement d'acquérir de nouvelles technologies."

... "L'investissement direct est l'un des moyens permettant une meilleure structuration des unités de production, d'une part, et une assimilation ainsi qu'une mise en cuvre plus efficaces des technologies nouvelles d'autre part. La nature et le volume des investissements directs dans un pays dépendent très largement de la politique menée par ses autorités, de la clarté et de la stabilité des règles juridiques applicables à ces investissements, de l'octroi du traitement national et du libre transfert des bénéfices ainsi que de la rentabilité probable de ces investissements, celle-ci étant fonction de la politique menée par le gouvernement et des structures économiques et sociales du pays. II est de la compétence de chaque Etat de déterminer en toute liberté la politique qu'il souhaite appliquer aux investissements étrangers, mais cette marge de manœuvre est plus limitée si cet Etat souhaite véritablement créer un climat favorable à l'investissement direct que la Suisse, de son côté, favorise dans les pays en développement, notamment par un réseau d'accords bilatéraux sur la protection des investissements et par un système de garantie des risques à l'investissement" (12).

Les 96 Etats qui participent actuellement aux négociations se sont mis d'accord sur quatre des dix chapitres qui forment le code : objectifs et principes (chapitre 2), réglementation nationale (chapitre 3), octroi d'un traitement spécial pour les pays en développement (chapitre 6), et collaboration internationale (chapitre 7).

L'objectif numéro un du code est d' "établir des normes générales et équitables qui serviront de base aux relations entre les parties aux transactions portant sur un transfert de technologie et les gouvernements intéressés, en prenant en considération leurs intérêts légitimes et en tenant dûment compte des besoins propres aux pays en développement en vue de la réalisation de leurs objectifs de développement économique et social" (13). Le code vise également à intensifier les transferts de technologie afin d'augmenter la participation des pays du Tiers Monde à la production et au commerce mondial, et à faciliter l'adoption et l'application de politiques nationales et à favoriser des transferts partiels. 
Quant aux principes, ils proclament la portée universelle du code, la souveraineté des Etats, et la prise en considération des objectifs de développement du pays acquéreur, mais aussi les avantages mutuels du fournisseur et de l'acquéreur et la protection des droits de propriété industrielle (chapitre 2).

La réglementation nationale (chapitre 3), dans le code, devrait favoriser les transferts de technologie et prendre en considération, de manière équitable, les intérêts légitimes de toutes les parties. $\mathrm{Ce}$ chapitre contient une liste de domaines dans lesquels les Etats peuvent prendre des mesures - financiers, techniques, de renégociation et d'organisation des transferts.

Tel que défini dans le Code, le traitement spécial des pays du Tiers Monde (chapitre 6) consiste principalement en une aide accrue des pays industrialisés, et à des conditions plus favorables pour l'implantation d'instituts de recherches scientifiques et technologiques dans le Tiers Monde. Les pays industrialisés devraient faciliter l'accès à l'information sur les caractéristiques et les coûts des technologies. Ce chapitre demande également aux pays industrialisés d'encourager les entreprises exportatrices à former leurs employés dans le Tiers Monde, à mettre au point une technologie adaptée, et à entreprendre dans le Tiers Monde une activité de recherche-développement.

La collaboration internationale (chapitre 7) vise à renforcer les capacités technologiques de tous les pays, par un plus grand échange d'information sur les technologies et sur les transferts - y compris sur les pratiques restrictives. Elle comprend également la promotion d'accords internationaux pour assurer un traitement équitable à l'acquéreur et au fournisseur de technologie, y compris des accords en vue d'éviter une double imposition.

Les négociateurs se sont également mis d'accord sur quatorze pratiques restrictives (chapitre 4). Parmi elles, on trouve les restrictions imposées à l'emploi, à la recherche, aux achats, aux ventes, aux prix, à l'adaptation de la technologie. Les pays industrialisés considèrent que cette liste est exhaustive. Les pays du Tiers Monde aimeraient inclure six autres pratiques restrictives (limitation à la production et au champ d'activité, contrôles de qualité inutiles, obligation d'utiliser une marque de fabrique, obligation de participer 
au capital, durée excessive de l'accord, limitation imposée à l'emploi d'une technologie déjà importée). De plus, le Groupe des 77 considère que les parties à un transfert de technologie "doivent" s'abstenir de pratiques restrictives. Quant au Groupe B, il estime que les parties ne "devraient" s'en abstenir que lorsque ces pratiques sont "abusives" ou "injustifiées". La Conférence doit maintenant trouver un mécanisme capable de déterminer quand il $y$ a abus.

Tous les Groupes se sont mis d'accord que le code concernait les transferts "internationaux". Cependant, pour échapper aux règles du code, une filiale pourrait transférer à une entreprise située dans le même pays la technologie de la société-mère. Les pays du Tiers Monde veulent étendre le champ d'application du code (chapitre 1) à ce type de transaction. Les pays industrialisés proposent de laisser les Etats libres d'appliquer le code aux transactions intérieures.

Un autre problème non résolu est celui des filiales elles-mêmes. Les pays industrialisés ont été d'accord de les inclure dans la définition des "parties" à un transfert de technologie. En contrepartie, ils ont demandé de pouvoir faire des réserves quant à l'application de certaines règles aux relations entre sociétés-mères et filiales. En fait, il s'agit d'autoriser certaines pratiques restrictives et de restreindre les responsabilités et les obligations dans les relations entre sociétés-mères et filiales (14).

Le projet de chapitre 5 sur les responsabilités et obligations des parties a été accepté par le Groupe B mais il a été rejeté en bloc par le Groupe des 77 et les Etats-Unis. Il sera donc entièrement renégocié. Les pays du Tiers Monde considèrent que le projet $n$ 'inclut pas les obligations fondamentales des parties. En outre, l'Organisation internationale du travail a fait un appel pour que le code contienne une disposition rendant responsable le fournisseur de technologie en cas de dommage infligé à des biens ou à des personnes et découlant de la technologie ou des biens produits. Cette disposition existait dans un projet antérieur mais elle a été supprimée dans le projet accepté par les pays industrialisés (15).

La question du droit applicable dans le règlement des différends (chapitre 9) est en suspens. Les pays industrialisés proposent que 
les parties liées par un transfert de technologie puissent choisir n'importe quel droit pour régler tous les problèmes qui surgiraient lors de la formation ou de l'exécution du contrat, et n'importe quel tribunal serait saisi en cas de différend à moins que ceci n'impose une lourde charge financière à l'une des parties. Ils proposent également que les tribunaux tiennent compte des usages commerciaux et que les gouvernements s'efforcent de favoriser les arrangements à l'amiable. Les pays du Tiers Monde estiment que le droit applicable aux questions d'ordre public et de souveraineté est celui du pays acquéreur. Ils proposent aussi que le droit applicable aux questions d'intérêt privé soit celui qui présente un lien direct, effectif et permanent avec le contrat. Ils proposent enfin, en cas de litige, le choix d'un arbitre venant d'un pays tiers selon une liste établie dans le code, et que le droit applicable soit interprété conformément au code.

Reste cette question centrale : il ne suffit pas seulement de terminer la rédaction de ce code, il s'agit également de savoir s'il représentera simplement un ensemble de principes que les Etats auront toute liberté de suivre ou s'il aura un caractère obligatoire engageant les Etats à modifier leur législation pour la rendre conforme au code (préambule du code).

Voici ce que dit le Conseil fédéral à ce propos : "Un pas important a été accompli dans la mesure où les pays en développement ont accepté que ce code soit maintenant conçu sous la forme de lignes directrices volontaires et non plus sous celle d'un traité international juridiquement contraignant. Ce progrès correspond à la position prise depuis le début des travaux par la Suisse et la plupart des autres pays développés occidentaux" (16).

Mais les 77 ont proposé qu'une conférence de révision ait lieu quatre ans après l'adoption du code et qu'elle décide de son caractère juridique (17). L'idée d'une révision a été acceptée par tous les groupes régionaux, mais plusieurs solutions intermédiaires sont encore à l'étude. Par exemple, le code pourrait contenir des règles obligatoires et des règles facultatives; ou bien il pourrait être accepté en tant que résolution par l'Assemblée générale des Nations Unies mais les Etats pourraient le ratifier indépendamment, totalement ou en partie; dans ce cas il aurait force obligatoire pour les signataires. 
Notes

1. $15 \mathrm{e}$ Rapport sur la politique économique extérieure, 13 août 1980 (FF 1980 III 1), p. 51.

2. Pour le texte du Code et les différentes propositions, voir : CNUCED : TD/CODE TOT/33, Projet de code international de conduite pour le transfert de technologie, 10 avril 1981 et CNUCED : TD/CODE TOT/25, Projet de code international de conduite pour le transfert de technologie, 6 mai 1980, Appendice $C$, texte se rapportant au chapitre 5 .

CNUCED : TD/CODE TOT/28, Négociation d'un code international de conduite pour le transfert de technologie, Présentation comparative de la position des groupes régionaux sur les principales questions en suspens, 23 mars 1981.

CNUCED : TD/CODE/27, Négociation d'un code international de conduite pour le transfert de technologie, Principales questions en suspens, 23 mars 1981.

Pour la position suisse, voir :

Conférence des Nations Unies sur la science et la technologie au service du développement : Document national suisse, 24 octobre 1978.

3. TOT 33, p. 1.

4. CNUCED : TD/CODE TOT/26, Choix de documents de la troisième session de la Conférence, 1980, SR.14, p. 6.

5. OCDE : Coopération pour le développement, Examen 1980, p. 89.

6. OCDE : Les enjeux du transfert de technologie Nord-Sud, 1981, pp. 36-37.

7. Office fédéral de la propriété intellectuelle : Rapport annuel 1980, pp. 24-25.

8. World Industrial Property Organization (WIPO) : Industrial Property Statistics 1979, Genève 1981, pp. 11, 13 et 15.

9. Richard Gerster: Patentierte Profite, Z-Verlag, Basel 1980, p. 42. 
10. OCDE : Communiqué de presse du 15 juin 1981 (A(81)26) : Les ressources mises à la disposition des pays en développement en 1980 et les tendances récentes.

11. CNUCED : TD/CODE TOT/27 Add. 1 : Négociation d'un code international de conduite pour le transfert de technologie, Principales étapes des travaux d'élaboration d'un code international de conduite pour le transfert de technologie, et Résolutions de base portant sur le code international de conduite pour le transfert de technologie.

12. Document suisse cité, pp. 20 et 28.

13. TOT 33, p. 4.

14. TOT 25, p. 1 note 3 et TOT 27, p. 14.

15. TOT 26 SR. 13, p. 5.

16. 14e Rapport sur la politique économique extérieure, 30 janvier 1980, p. 53.

17. TOT 27, pp. 10-11. 